\title{
Avoidance colourings for small nonclassical Ramsey numbers
}

\author{
AP Burger and JH van Vuuren \\ Department of Logistics, University of Stellenbosch, Private Bag X1, Matieland, 7602, South Africd \\ received $1^{\text {st }}$ April 2010, revised 16 $6^{\text {th }}$ May 2011, accepted 13 $3^{\text {th }}$ June 2011.
}

\begin{abstract}
The irredundant Ramsey number $s=s(m, n)$ [upper domination Ramsey number $u=u(m, n)$, respectively] is the smallest natural number $s$ [u, respectively] such that in any red-blue edge colouring $(R, B)$ of the complete graph of order $s$ [u, respectively], it holds that $\operatorname{IR}(B) \geq m$ or $I R(R) \geq n[\Gamma(B) \geq m$ or $\Gamma(R) \geq n$, respectively], where $\Gamma$ and $I R$ denote respectively the upper domination number and the irredundance number of a graph. Furthermore, the mixed irredundant Ramsey number $t=t(m, n)$ [mixed domination Ramsey number $v=v(m, n)$, respectively] is the smallest natural number $t$ [ $v$, respectively] such that in any red-blue edge colouring $(R, B)$ of the complete graph of order $t$ [ $v$, respectively], it holds that $I R(B) \geq m$ or $\beta(R) \geq n[\Gamma(B) \geq m$ or $\beta(R) \geq n$, respectively], where $\beta$ denotes the independent domination number of a graph. These four classes of non-classical Ramsey numbers have previously been studied in the literature. In this paper we introduce a new Ramsey number $w=w(m, n)$, called the irredundant-domination Ramsey number, which is the smallest natural number $w$ such that in any red-blue edge colouring $(R, B)$ of the complete graph of order $w$, it holds that $I R(B) \geq m$ or $\Gamma(R) \geq n$. A computer search is employed to determine complete sets of avoidance colourings of small order for these five classes of nonclassical Ramsey numbers. In the process the fifteen previously unknown Ramsey numbers $t(4,4)=14, t(6,3)=17$, $u(4,4)=13, v(4,3)=8, v(4,4)=14, v(5,3)=13, v(6,3)=17, w(3,3)=6, w(3,4)=w(4,3)=8$, $w(4,4)=13, w(3,5)=w(5,3)=12$ and $w(3,6)=w(6,3)=15$ are established.
\end{abstract}

Keywords: Mixed Ramsey number, nonclassical Ramsey number, extremal colouring.

\section{Introduction}

A bi-colouring of the edges of a graph, using the colours red and blue, is called a red-blue edge colouring of the graph. If $R$ and $B$ are the subgraphs induced by respectively the red and the blue edges of such a colouring, the colouring is denoted by the ordered pair $(R, B)$, and $R$ is referred to as the red subgraph, while $B$ is called the blue subgraph. For any natural numbers $m$ and $n$, the (classical) Ramsey number $r=r(m, n)$ is the smallest natural number $r$ such that, for any red-blue edge colouring $(R, B)$ of the complete graph $K_{r}$ on $r$ vertices, it holds that either $K_{m} \subseteq R$ or $K_{n} \subseteq B$, or perhaps both.

\footnotetext{
†emails: apburgeresun.ac.za and vuurenesun.ac.za

1365-8050 @ 2011 Discrete Mathematics and Theoretical Computer Science (DMTCS), Nancy, France
} 
The notion of a Ramsey number may also be defined in terms of independent sets in graphs. A subset $\mathcal{I}$ of vertices of a graph $G$ is said to be independent if no two vertices in $\mathcal{I}$ are adjacent in $G$. The cardinality of a largest independent set of $G$ is denoted by $\beta(G)$, called the independent domination number of $G$. If, for some red-blue edge colouring $(R, B)$, it holds that $K_{m} \subseteq R$, then there is an independent set of order $m$ in $B$, and hence $\beta(B) \geq m$. Similarly, $K_{n} \subseteq B$ implies that $\beta(R) \geq n$. The classical Ramsey number $r(m, n)$ may therefore be redefined as the smallest natural number $r$ such that in any red-blue edge colouring $(R, B)$ of the complete graph $K_{r}$ of order $r$, it holds that $\beta(B) \geq m$ or $\beta(R) \geq n$ (or perhaps both). For this reason the classical Ramsey number $r(m, n)$ is often referred to as the independent Ramsey number. This definition has been generalised in many ways. Four generalisations that arose during the period 1989-1992 involve the so-called upper domination number and the irredundance number of a graph - parameters that are closely related to the independent domination number of a graph.

A subset $\mathcal{D}$ of the vertices of a graph $G$ is said to be dominating if every vertex $v$ of $G$ is an element of $\mathcal{D}$ or is adjacent in $G$ to an element of $\mathcal{D}$. A dominating set $\mathcal{D}$ of $G$ is said to be minimal if no proper subset of $\mathcal{D}$ is a dominating set in $G$. The cardinality of a largest minimal dominating set of $G$ is denoted by $\Gamma(G)$, called the upper domination number of $G$.

The closed neighbourhood of a graph vertex $v$, denoted by $N[v]$, is defined as the subset of all vertices of $G$ that are adjacent to $v$ in $G$, together with the vertex $v$ itself. The closed neighbourhood of a set of vertices $\mathcal{S}=\left\{v_{1}, \ldots, v_{m}\right\}$ in $G$, denoted by $N[\mathcal{S}]$, is simply defined as $N[\mathcal{S}]=\cup_{i=1}^{m} N\left[v_{i}\right]$. The set of private neighbours of a vertex $v \in \mathcal{V}_{G}$ with respect to some subset $\mathcal{S} \subset \mathcal{V}_{G}$ is defined as $\operatorname{PN}(v, \mathcal{S})=$ $N[v] \backslash N[\mathcal{S} \backslash\{v\}]$. A subset $\mathcal{X}$ of the vertices of $G$ is said to be irredundant if every vertex $v \in \mathcal{X}$ has at least one private neighbour in $G$ with respect to $\mathcal{X}$ (that is, if $\operatorname{PN}(v, \mathcal{X}) \neq \emptyset$ for all $v \in \mathcal{X}$ ). Finally, the cardinality of a largest maximal irredundant set of $G$ is denoted by $\operatorname{IR}(G)$, called the irredundance number of $G$.

It holds for any graph $G$ that

$$
\beta(G) \leq \Gamma(G) \leq \operatorname{IR}(G) .
$$

This result dates back to 1978 and is due to Cockayne et al. [4]. The following four so-called nonclassical Ramsey numbers have previously been studied in the literature:

The irredundant Ramsey number $s=s(m, n)$ is the smallest natural number $s$ such that in any red-blue edge colouring $(R, B)$ of the complete graph $K_{s}$ of order $s$, it holds that $I R(B) \geq m$ or $I R(R) \geq n$ (or perhaps both). This definition is due to Brewster et al. [1].

The mixed irredundant Ramsey number $t=t(m, n)$ is the smallest natural number $t$ such that in any red-blue edge colouring $(R, B)$ of the complete graph $K_{t}$ of order $t$, it holds that $I R(B) \geq m$ or $\beta(R) \geq n$ (or perhaps both). This definition is due to Cockayne et al. [5].

The upper domination Ramsey number $u=u(m, n)$ is the smallest natural number $u$ such that in any red-blue edge colouring $(R, B)$ of the complete graph $K_{u}$ of order $u$, it holds that $\Gamma(B) \geq m$ or $\Gamma(R) \geq n$ (or perhaps both). This definition is due to Oellermann and Shreve [18].

The mixed domination Ramsey number $v=v(m, n)$ is the smallest natural number $v$ such that in any red-blue edge colouring $(R, B)$ of the complete graph $K_{v}$ of order $v$, it holds that $\Gamma(B) \geq m$ or $\beta(R) \geq n$ (or perhaps both). This definition is also due to Oellermann and Shreve [18].

Finally, the following Ramsey number seems not to have been considered previously: 
The irredundant-domination Ramsey number $w=w(m, n)$ is the smallest natural number $w$ such that in any red-blue edge colouring $(R, B)$ of the complete graph $K_{w}$ of order $w$, it holds that $I R(B) \geq m$ or $\Gamma(R) \geq n$ (or perhaps both).

The arguments of the classical, irredundant and upper domination Ramsey numbers may be interchanged without altering the values of these numbers, i.e. $r(m, n)=r(n, m), s(m, n)=s(n, m)$ and $u(m, n)=u(n, m)$ for any integers $m, n \geq 2$. However, in the case of the mixed Ramsey numbers $t(m, n), v(m, n)$ and $w(m, n)$, the arguments $m$ and $n$ do not necessarily commute, i.e. $t(m, n) \neq$ $t(n, m), v(m, n) \neq v(n, m)$ and $w(m, n) \neq w(n, m)$ in general. Furthermore, the Ramsey numbers defined above are related to each other and to the classical Ramsey number $r(m, n)$ by means of the inequality chains

$$
s(m, n) \leq w(m, n) \leq\left\{\begin{array}{c}
u(m, n) \\
t(m, n)
\end{array}\right\} \leq v(m, n) \leq r(m, n)
$$

which are easily shown to hold for all $m, n \geq 2$ in view of (1). It is unknown in what way (if any) the Ramsey numbers $u(m, n)$ and $t(m, n)$ are related.

The well-known recursive upper bounds

$$
\begin{aligned}
r(m, n) & \leq r(m-1, n)+r(m, n-1) \\
s(m, n) & \leq s(m-1, n)+s(m, n-1) \\
\text { and } t(m, n) & \leq t(m-1, n)+t(m, n-1)
\end{aligned}
$$

hold, and any one of these inequalities is strict if both terms on the right hand side are even.

To the best knowledge of the authors, the numbers listed in Table 1 are the only known nonclassical Ramsey numbers and the bounds listed in the table are the best currently available. Using the inequality chains 2 it is possible to derive the values of and bounds on the Ramsey numbers $w(m, n)$ shown in Table 2 from the corresponding Ramsey number values in Table 1.

$$
\begin{aligned}
& \begin{array}{ccccccc}
s(2, n) & s(3,3) & s(3,4) & s(3,5) & s(3,6) & s(3,7) & s(4,4) \\
\hline n & 6^{a} & 8^{a} & 12^{a} & 15^{b} & 18^{c} & 13^{d}
\end{array} \\
& \begin{array}{cccccccccc}
t(2, n) & t(n, 2) & t(3,3) & t(3,4) & t(4,3) & t(3,5) & t(5,3) & t(3,6) & t(6,3) & t(4,4) \\
\hline n & n & 6^{e} & 9^{e} & 8^{e} & 12^{e} & 13^{e} & 15^{f} & 15: 18^{g} & 13: 17^{g}
\end{array} \\
& \begin{array}{ccccccc}
u(2, n) & u(3,3) & u(3,4) & u(3,5) & u(3,6) & u(3,7) & u(4,4) \\
\hline n & 6^{h} & 8^{h} & 12^{h} & 15^{h} & 18: 22^{g} & 13: 15^{h}
\end{array} \\
& \begin{array}{cccccccccc}
v(2, n) & v(n, 2) & v(3,3) & v(3,4) & v(4,3) & v(3,5) & v(5,3) & v(3,6) & v(6,3) & v(4,4) \\
\hline n & n & 6^{i} & 9^{i} & 8: 9^{j} & 12^{i} & 13: 14^{j} & 15^{i} & 15: 18^{g} & 13: 18^{g}
\end{array}
\end{aligned}
$$

Tab. 1: Values of and bounds on nonclassical Ramsey numbers from the literature. Bounds are given in the format [lower bound : upper bound] (inclusive). ${ }^{a}$ Due to Brewster et al. [1], ${ }^{b}$ Due to Brewster et al. [2], ${ }^{c}$ Due to Cockayne et al. [6] as well as Chen and Rousseau [3], ${ }^{d}$ Due to Cockayne et al. [7], ${ }^{e}$ Due to Cockayne et al. [5], ${ }^{f}$ Due to Grobler [11], ${ }^{g}$ By the inequalities [2]-[5], ${ }^{h}$ Due to Dzido and Zakrzewska [8], ${ }^{i}$ Due to Henning and Oellermann [15], ${ }^{j}$ Due to Henning and Oellermann [14]. 


\begin{tabular}{cccccccccc}
$w(2, n)$ & $w(n, 2)$ & $w(3,3)$ & $w(3,4)$ & $w(4,3)$ & $w(3,5)$ & $w(5,3)$ & $w(3,6)$ & $w(6,3)$ & $w(4,4)$ \\
\hline$n$ & $n$ & 6 & 8 & 8 & 12 & 12 & 15 & 15 & $13: 15$
\end{tabular}

Tab. 2: Values of and bounds on the nonclassical Ramsey number $w(m, n)$, as derived from the entries in Table 1 in view of the inequality chains $(2)$.

For any integer $q<s(m, n)$, a red-blue edge colouring $(R, B)$ of the complete graph $K_{q}$ for which

$$
\operatorname{IR}(B)<m \text { and } \operatorname{IR}(R)<n
$$

is called an avoidance $s(m, n)$-colouring. The two inequalities in (6) are called avoidance conditions associated with the Ramsey number $s(m, n)$. Furthermore, an avoidance $s(m, n)$-colouring is said to be extremal if $q=s(m, n)-1$. (Extremal) avoidance $t(m, n)-, u(m, n)-, v(m, n)$ - and $w(m, n)$-colourings are defined similarly. The degree restrictions below hold for any avoidance $s(m, n)$-colouring and are due to Hattingh [12].

Theorem 1 Let $m, n>2$ be integers. Then, $\Delta(R)<s(m-1, n)$ and $\Delta(B)<s(m, n-1)$ in any avoidance $s(m, n)$-colouring $(R, B)$.

The following corollary therefore holds by (2).

Corollary 1 Let $m, n \geq 2$ be integers and let $x \in\{s, t, u, v, w\}$ be a nonclassical Ramsey number. Then, $\Delta(R)<x(m-1, n)$ and $\Delta(B)<x(m, n-1)$ in any avoidance $x(m, n)$-colouring $(R, B)$.

Finally, the following useful corollary immediately follows from Corollary 1.

Corollary 2 Let $m, n \geq 2$ be integers and let $x \in\{s, t, u, v, w\}$ be a nonclassical Ramsey number. Then, $\delta(R) \geq p-x(m, n-1)$ and $\delta(B) \geq p-x(m-1, n)$ in any avoidance $x(m, n)$-colouring $(R, B)$ of the complete graph of order $p$.

The objective in this paper is to determine the values of the outstanding nonclassical Ramsey numbers in Table 1 and to describe all the extremal avoidance colourings related to the Ramsey numbers in the table. The paper is organised as follows. We show analytically in $\S 2$ that unique avoidance colourings are associated with each of the Ramsey numbers $x(2, n), x(n, 2)$ and $x(3,3)$, where $x \in\{s, t, u, v, w\}$. In $\S 3$ we then describe a recursive algorithmic procedure for computing the values of the parameters $\beta(G)$, $\Gamma(G)$ and $\operatorname{IR}(G)$ of a small graph $G$. This procedure is applied in $\S 4$ to search for (extremal) avoidance colourings associated with small Ramsey numbers. In $\S 5$ it is shown that the search space may be reduced by considering only complete sets of avoidance colourings for the classical Ramsey number $r(m, n)-$ this reduction renders the search procedure of $\S 4$ feasible for uncovering (extremal) avoidance colourings associated with larger Ramsey numbers. We close, in $\S 6$, with a summary of the contributions in this paper. 


\section{The Ramsey numbers $x(2, n)$ and $x(3,3), x \in\{s, t, u, v, w\}$}

It is easy to establish the extremal avoidance colourings associated with the Ramsey numbers $x(2, n)=n$ and $x(n, 2)=n$, where $x \in\{s, t, u, v, w\}$.

Theorem 2 The red-blue edge colouring $(R, B)$ of the complete graph $K_{n-1}$ such that $R \cong \bar{K}_{n-1}$ and $B \cong K_{n-1}$ is the only extremal avoidance colouring associated with each of the Ramsey numbers $x(2, n)$, while the reverse edge bi-colouring $(B, R)$ of $K_{n-1}$ is the only extremal avoidance colouring associated with each of the Ramsey numbers $x(n, 2)$, where $x \in\{s, t, u, v, w\}$.

Proof: Let $\mathcal{C}_{1}=(R, B)$ be the red-blue edge colouring of the complete graph $K_{n-1}$ for which $R \cong$ $\bar{K}_{n-1}$ and $B \cong K_{n-1}$. Then clearly $\beta(B)=\Gamma(B)=I R(B)=1<2$ and $\beta(R)=\Gamma(R)=\operatorname{IR}(R)=$ $n-1<n$, so that $\mathcal{C}_{1}$ is indeed an extremal avoidance colouring associated with any one of the Ramsey numbers $x(2, n), x \in\{s, t, u, v, w\}$. Similarly, for the reverse red-blue edge colouring $\overline{\mathcal{C}}_{1}$ of $K_{n-1}$ (i.e. where $R \cong K_{n-1}$ and $B \cong \bar{K}_{n-1}$ ) it holds that $\beta(B)=\Gamma(B)=I R(B)=n-1<n$ and $\beta(R)=\Gamma(R)=\operatorname{IR}(R)=1<2$, so that $\overline{\mathcal{C}}_{1}$ is indeed an extremal avoidance colouring associated with any one of the Ramsey numbers $x(n, 2), x \in\{s, t, u, v, w\}$.

Conversely, let $\mathcal{C}_{2}=\left(R^{\prime}, B^{\prime}\right)$ be an extremal avoidance colouring associated with any one of the Ramsey numbers $x(2, n), x \in\{s, t, u, v, w\}$. Then one of the inequalities $\beta\left(B^{\prime}\right)<2, \Gamma\left(B^{\prime}\right)<2$ or $\operatorname{IR}\left(B^{\prime}\right)<2$ must hold. In any one of these cases $B^{\prime}$ must therefore be complete (since the omission of an edge in $B^{\prime}$ would imply the existence of an independent set, a minimal dominating set and an irredundant set of cardinality at least 2 in $\left.B^{\prime}\right)$, and hence $\mathcal{C}_{2}=\mathcal{C}_{1}$. Similarly, if $\mathcal{C}_{3}=\left(R^{\prime \prime}, B^{\prime \prime}\right)$ is an extremal avoidance colouring associated with any one of the Ramsey numbers $x(n, 2), x \in\{s, t, u, v, w\}$, then one of the inequalities $\beta\left(R^{\prime \prime}\right)<2, \Gamma\left(R^{\prime \prime}\right)<2$ or $I R\left(R^{\prime \prime}\right)<2$ must hold, in which case $R^{\prime \prime}$ must again be complete for the same reason as above. Hence $\mathcal{C}_{3}=\overline{\mathcal{C}}_{1}$.

It is also possible to show that a unique extremal avoidance colouring is associated with each of the Ramsey numbers $x(3,3), x \in\{s, t, u, v, w\}$.

Theorem 3 The red-blue edge colouring $(R, B)$ of the complete graph $K_{5}$ of order 5 for which $R \cong C_{5}$ and $B \cong C_{5}$ is the only extremal avoidance colouring associated with each of the Ramsey numbers $s(3,3)=t(3,3)=u(3,3)=v(3,3)=w(3,3)=6$.

Proof: Let $\mathcal{C}_{1}=(R, B)$ be the red-blue edge colouring of the complete graph $K_{5}$ for which $R \cong C_{5}$ and $B \cong C_{5}$. Then clearly $\beta(B)=\Gamma(B)=I R(B)=2<3$ and $\beta(R)=\Gamma(R)=I R(R)=2<3$, so that $\mathcal{C}_{1}$ is indeed an extremal avoidance colouring associated with any one of the Ramsey numbers $x(3,3)=6$, $x \in\{s, t, u, v, w\}$.

Conversely, let $\mathcal{C}_{2}=\left(R^{\prime}, B^{\prime}\right)$ be any extremal avoidance colouring associated with any one of the Ramsey numbers $x(3,3)=6, x \in\{s, t, u, v, w\}$. It follows by Corollaries 1 and 2 that every vertex in $R^{\prime}$ and every vertex in $B^{\prime}$ has degree 2 . But then $R^{\prime}$ and $B^{\prime}$ are both cycles, in which case they must both be isomorphic to $C_{5}$, since $R^{\prime}$ and $B^{\prime}$ are both of order 5 . 


\section{Computing the graph parameters $\beta, \Gamma$ and $I R$}

In this section we describe our recursive algorithmic approach towards computing the parameters $\beta(G)$, $\Gamma(G)$ and $I R(G)$ for a given graph $G$ in order to compute avoidance colourings for the Ramsey numbers in Table 1. Although the discussion in this section is mainly for the parameter $\operatorname{IR}(G)$, the other two graph parameters were computed in a similar manner, as we outline at the end of the section.

A pseudocode listing of our recursive Boolean approach towards determining whether or not a given subset of vertices $S$ of a labelled graph $G$ with vertex set $\left\{v_{0}, \ldots, v_{n-1}\right\}$ may be augmented (i.e. enlarged) and still remain irredundant in $G$ is given in Algorithm 1 . The algorithm takes the set $S$ as input and produces the Boolean value True as output if an irredundant set $S \cup T$ in $G$ of cardinality $\Psi$ can be found such that the index of every vertex in $T$ is larger than the largest index of any vertex in $S$, or False otherwise, where $\Psi$ is any valid upper bound on the parameter $\operatorname{IR}(G)$. The algorithm generates a branching-search tree by branching on the inclusion of all possible vertices $v_{i}$ with indices $i$ larger than the largest vertex index in $S$, calling itself with the set $S \cup\left\{v_{i}\right\}$ as input (instead of the original set $S$ ) at each branch.

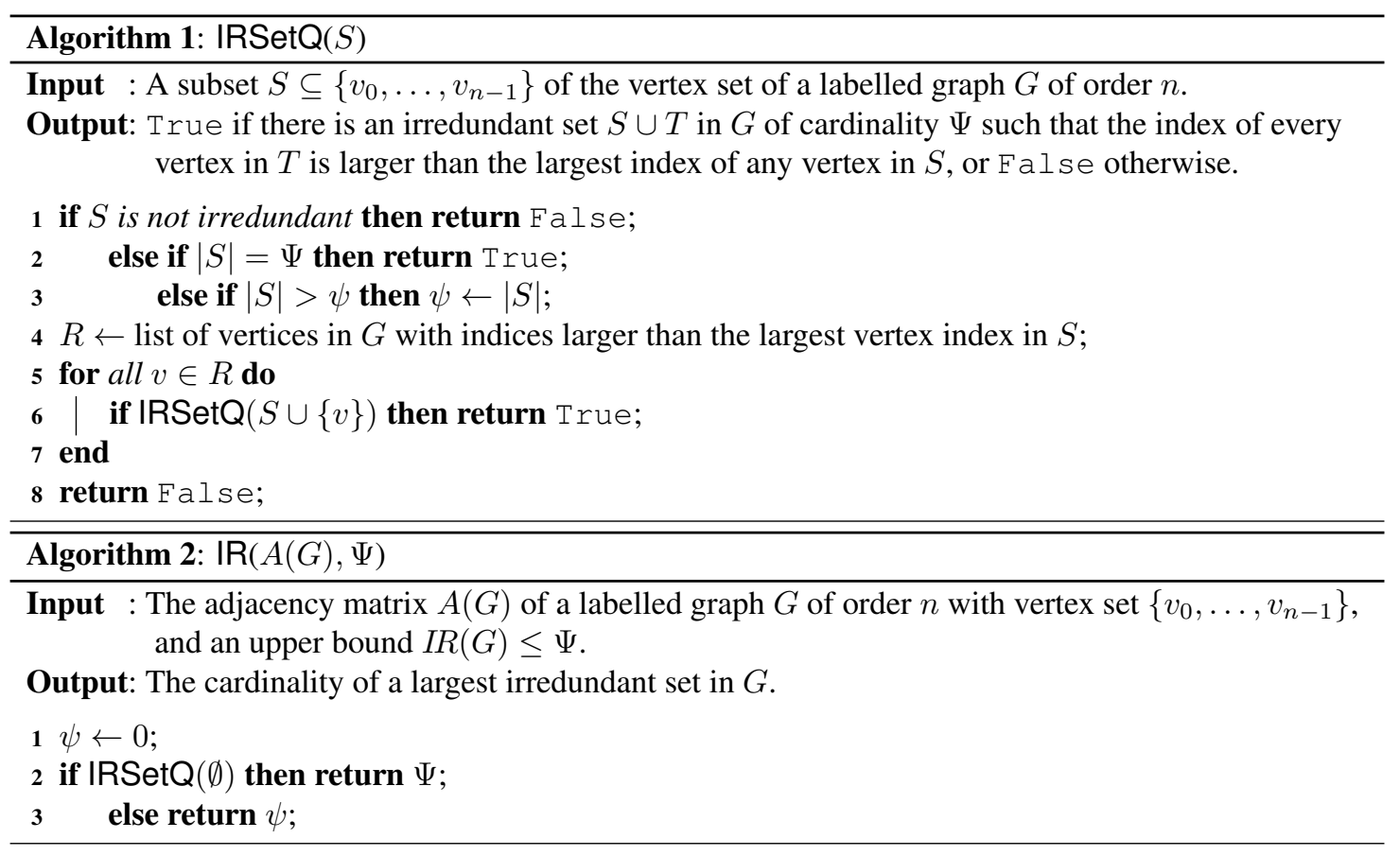

The root of the search tree is generated with a call to Algorithm 1 in which $S=\emptyset$. This call occurs in Step 2 of Algorithm 2. The input to Algorithm 2 is the adjacency matrix $A(G)$ of the graph $G$ as well as a user-specified upper bound $\Psi$ on the value of $\operatorname{IR}(G)$; both these input parameters are defined globally outside the scope of the various calls to the recursive algorithm IRSetQ $(S)$ and are never altered during construction of the search tree. Algorithm 2 yields as output the exact value of $\operatorname{IR}(G)$, employing a lower bound on the value of $\operatorname{IR}(G)$, which is initially taken as zero (Step 1 of Algorithm 2). This lower bound 
is updated globally as larger and larger irredundant sets are encountered in the search tree.

Computation of the parameter $\beta(G)$ occurs exactly as in the case of $\operatorname{IR}(G)$, the only change being replacement of the requirement that $S$ "is not irredundant" in Step 1 of Algorithm 1 by the requirement that $S$ "is not independent". However, care should be taken with the computation of the parameter $\Gamma(G)$, since it is not the cardinality of a largest dominating set in $G$, but of a largest minimal dominating set in $G$. However, it is well known that a dominating set is minimal if and only if it is dominating and irredundant [13. Proposition 3.8]. Hence the computation of the parameter $\Gamma(G)$ proceeds exactly as in the case of $I R(G)$, the only changes being replacement of the requirement that " $|S|=\Psi$ " in Step 2 of Algorithm 1 by the requirement that " $|S|=\Psi$ and $S$ is dominating," and replacement of the requirement that " $|S|>\psi$ " in Step 3 by the requirement that " $|S|>\psi$ and $S$ is dominating."

\section{Ramsey numbers not exceeding the value 9}

A natural, brute-force approach towards determining a complete set of extremal avoidance colourings of a small Ramsey number $x(m, n)$ in Table 1, where $x \in\{s, t, u, v, w\}$, is to step through each of the nonisomorphic unlabelled graphs of order $x(m, n)-1$ and to test, for each possible graph $R$ and its graph complement $B$, whether or not both avoidance conditions associated with the Ramsey number $x(m, n)$ are satisfied by the edge colouring $(R, B)$. Such a brute-force approach is facilitated by repositories of the various non-isomorphic graph structures of small orders, such as the one on McKay's website [16]. By adopting such a brute-force search involving all 1044 graphs of order 7 it is possible to prove the following theorem.

Theorem $4 v(4,3)=8$.

Taking the nine graphs $E_{1}-E_{9}$ in Figure 1(a)-(i) as blue subgraphs produces complete sets of extremal avoidance $v(4,3)$-colourings and extremal avoidance $t(4,3)$-colourings. The above exhaustive search also shows that taking the eight graphs $E_{1}-E_{8}$ in Figure 1 (a)-(h) as red subgraphs produces complete sets of extremal avoidance $s(3,4)$-colourings, extremal avoidance $u(3,4)$-colourings and extremal avoidance $w(3,4)$-colourings. However, taking the eight graphs $E_{1}-E_{8}$ in Figure 1 (a)-(h) as blue subgraphs produces a complete set of extremal avoidance $w(4,3)$-colourings.

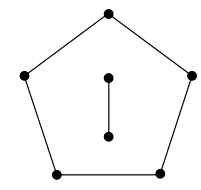

(a) $E_{1}$

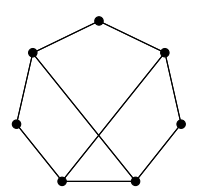

(g) $E_{7}$

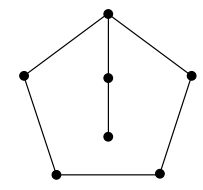

(b) $E_{2}$

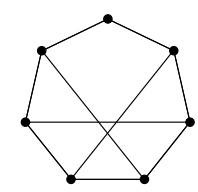

(h) $E_{8}$

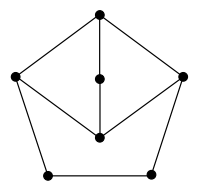

(c) $E_{3}$

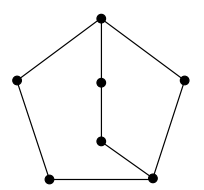

(i) $E_{9}$

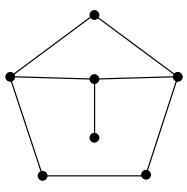

(d) $E_{4}$

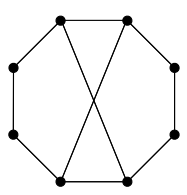

(j) $E_{10}$

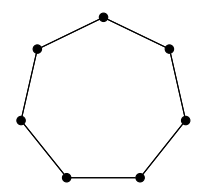

(e) $E_{5}$

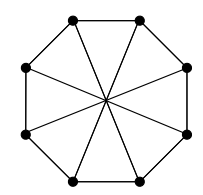

(k) $E_{11}$

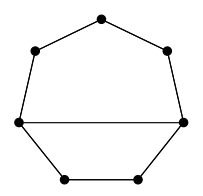

(f) $E_{6}$

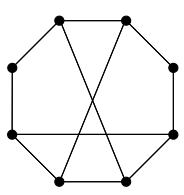

(1) $E_{12}$

Fig. 1: Extremal avoidance graphs of orders 7 and 8. 
We also immediately have the following corollary from Theorem 4 .

Corollary $3 v(5,3)=13$.

Proof: Suppose that an avoidance $v(5,3)$-colouring $(R, B)$ of $K_{13}$ exists. Then it follows by Theorem 4 and Corollaries 1 th that $\delta(R) \geq 13-v(5,2)=8$ but that $\Delta(R)<v(4,3)=8$. This contradiction shows that no avoidance $v(5,3)$-colouring of $K_{13}$ exists and hence that $v(5,3) \leq 13$, yielding the desired result by Table 1 .

Finally, an exhaustive search involving all 12346 graphs of order 8 shows that taking the two graphs $E_{10}$ and $E_{11}$ in Figure $1(\mathrm{j})-(\mathrm{k})$ as red subgraphs produces complete sets of extremal avoidance $t(3,4)$ colourings and extremal avoidance $v(3,4)$-colourings, while it is known that taking the three graphs $E_{10^{-}}$ $E_{12}$ in Figure $1 \mathrm{j}$ j)-(1) as red subgraphs produces a complete set of extremal avoidance $r(3,4)$-colourings [16].

Unfortunately the brute-force search approach described above is only feasible for small Ramsey numbers. In fact, we were only able to implement the brute-force search approach described above for cases where the relevant Ramsey number does not exceed the value 9, in view of the rapid growth of the number of non-isomorphic graphs of order $n$ as $n$ increases. A reduced search space is therefore required in order to find a complete set of extremal avoidance colourings for Ramsey numbers larger than 9 .

We close this section by remarking that although the brute-force search approach considered here is very primitive and certainly not interesting of its own accord, it is nevertheless a useful validation mechanism for results uncovered by other search techniques.

\section{Computing avoidance colourings from the classical case}

A search technique capable of finding complete sets of avoidance colourings for larger Ramsey numbers stems from the following result which states that avoidance colourings are transferred from left to right between the Ramsey numbers in the inequality chains (2).

Theorem 5 Let $m, n>2$ be integers, and let $x(m, n)$ and $y(m, n)$ be any two Ramsey numbers in (2) satisfying $x(m, n) \leq y(m, n)$. Then any avoidance $x(m, n)$-colouring is also an avoidance $y(m, n)$ colouring.

Proof: Let $(R, B)$ be an avoidance $x(m, n)$-colouring. Then $\xi_{x}(B)<m$ and $\zeta_{x}(R)<n$, where $\xi_{x}$ and $\zeta_{x}$ are the parameters in 11 that are relevant to the Ramsey number $x(m, n)$. Since $x(m, n) \leq y(m, n)$ in (2), it follows that $\xi_{y}(B) \leq \xi_{x}(B)$ and $\zeta_{y}(B) \leq \zeta_{x}(B)$ in 11 , so that $(R, B)$ is also an avoidance $y(m, n)$-colouring.

The significance of Theorem 5 is that we do not have to consider all possible colourings, but rather only those for the classical Ramsey number $r(m, n)$ when seeking avoidance colourings for the nonclassical numbers $s(m, n), t(m, n), u(m, n), v(m, n)$ or $w(m, n)$ by virtue of the inequality chains 22. McKay [17] provides complete sets of avoidance colourings of all orders $p<r(m, n)$ for the small classical Ramsey numbers $r(3,3)=6, r(3,4)=9, r(3,5)=14, r(3,6)=18$ and $r(4,4)=18$. By considering each of these avoidance colourings and testing via the algorithmic approach described in $\S 3$ in which cases the avoidance conditions associated with the nonclassical Ramsey numbers are satisfied, we were able to establish the cardinalities of complete sets of avoidance colourings shown in Tables 3 .4. The values in 


\begin{tabular}{r|rrrrrr}
$p$ & $s(3,3)$ & $w(3,3)$ & $u(3,3)$ & $t(3,3)$ & $v(3,3)$ & $r(3,3)$ \\
\hline 1 & 1 & 1 & 1 & 1 & 1 & 1 \\
2 & 2 & 2 & 2 & 2 & 2 & 2 \\
3 & 2 & 2 & 2 & 2 & 2 & 2 \\
4 & 3 & 3 & 3 & 3 & 3 & 3 \\
5 & $\underline{1}$ & $\underline{1}$ & $\underline{1}$ & $\underline{1}$ & $\underline{1}$ & $\underline{1}$
\end{tabular}

\begin{tabular}{r||rrrrrr|rrr}
$p$ & $s(3,4)$ & $w(3,4)$ & $u(3,4)$ & $t(3,4)$ & $v(3,4)$ & $r(3,4)$ & $w(4,3)$ & $t(4,3)$ & $v(4,3)$ \\
\hline 1 & 1 & 1 & 1 & 1 & 1 & 1 & 1 & 1 & 1 \\
2 & 2 & 2 & 2 & 2 & 2 & 2 & 2 & 2 & 2 \\
3 & 3 & 3 & 3 & 3 & 3 & 3 & 3 & 3 & 3 \\
4 & 6 & 6 & 6 & 6 & 6 & 6 & 6 & 6 & 6 \\
5 & 9 & 9 & 9 & 9 & 9 & 9 & 9 & 9 & 9 \\
6 & 14 & 14 & 14 & 14 & 14 & 15 & 14 & 15 & 15 \\
7 & $\underline{8}$ & $\underline{8}$ & $\underline{8}$ & 8 & 8 & 9 & $\underline{8}$ & $\underline{9}$ & $\underline{9}$ \\
8 & 0 & 0 & 0 & $\underline{2}$ & $\underline{2}$ & $\underline{3}$ & 0 & 0 & 0
\end{tabular}

\begin{tabular}{l||rrrrrr|rrr}
$p$ & $s(3,5)$ & $w(3,5)$ & $u(3,5)$ & $t(3,5)$ & $v(3,5)$ & $r(3,5)$ & $w(5,3)$ & $t(5,3)$ & $v(5,3)$ \\
\hline 1 & 1 & 1 & 1 & 1 & 1 & 1 & 1 & 1 & 1 \\
2 & 2 & 2 & 2 & 2 & 2 & 2 & 2 & 2 & 2 \\
3 & 3 & 3 & 3 & 3 & 3 & 3 & 3 & 3 & 3 \\
4 & 7 & 7 & 7 & 7 & 7 & 7 & 7 & 7 & 7 \\
5 & 13 & 13 & 13 & 13 & 13 & 13 & 13 & 13 & 13 \\
6 & 31 & 31 & 31 & 31 & 31 & 32 & 31 & 32 & 32 \\
7 & 66 & 66 & 66 & 66 & 66 & 71 & 66 & 71 & 71 \\
8 & 147 & 147 & 148 & 147 & 148 & 179 & 148 & 179 & 179 \\
9 & 184 & 184 & 185 & 186 & 187 & 290 & 185 & 287 & 287 \\
10 & 69 & 69 & 70 & 93 & 94 & 313 & 70 & 221 & 221 \\
11 & 1 & $\underline{1}$ & $\underline{1}$ & $\underline{6}$ & $\underline{6}$ & 105 & 1 & 16 & 16 \\
12 & 0 & 0 & 0 & 0 & 0 & 12 & 0 & $\underline{1}$ & $\underline{1}$ \\
13 & 0 & 0 & 0 & 0 & 0 & 1 & 0 & 0 & 0
\end{tabular}

\begin{tabular}{c||rrrrrr|rrr}
$p$ & $s(3,6)$ & $w(3,6)$ & $u(3,6)$ & $t(3,6)$ & $v(3,6)$ & $r(3,6)$ & $w(6,3)$ & $t(6,3)$ & $v(6,3)$ \\
\hline 1 & 1 & 1 & 1 & 1 & 1 & 1 & 1 & 1 & 1 \\
2 & 2 & 2 & 2 & 2 & 2 & 2 & 2 & 2 & 2 \\
3 & 3 & 3 & 3 & 3 & 3 & 3 & 3 & 3 & 3 \\
4 & 7 & 7 & 7 & 7 & 7 & 7 & 7 & 7 & 7 \\
5 & 14 & 14 & 14 & 14 & 14 & 14 & 14 & 14 & 14 \\
6 & 36 & 36 & 36 & 36 & 36 & 37 & 36 & 37 & 37 \\
7 & 95 & 95 & 95 & 95 & 95 & 100 & 95 & 100 & 100 \\
8 & 311 & 311 & 312 & 311 & 312 & 356 & 312 & 356 & 356 \\
9 & 1058 & 1058 & 1065 & 1058 & 1065 & 1407 & 1065 & 1407 & 1407 \\
10 & 3680 & 3680 & 3738 & 3682 & 3740 & 6657 & 3738 & 6654 & 6654 \\
11 & 9306 & 9306 & 9550 & 9341 & 9585 & 30395 & 9550 & 30275 & 30275 \\
12 & 9464 & 9464 & 9887 & 10277 & 10705 & 116792 & 9887 & 106701 & 106701 \\
13 & 1167 & 1167 & 1245 & 2039 & 2135 & 275086 & 1245 & 155472 & 155472 \\
14 & 2 & 2 & $\underline{2}$ & $\underline{34}$ & $\underline{37}$ & 263520 & 2 & 39049 & 39049 \\
15 & 0 & 0 & 0 & 0 & 0 & 64732 & 0 & 508 & 508 \\
16 & 0 & 0 & 0 & 0 & 0 & 2576 & 0 & $\underline{2}$ & $\underline{2}$ \\
17 & 0 & 0 & 0 & 0 & 0 & $\underline{7}$ & 0 & 0 & 0
\end{tabular}

Tab. 3: Cardinalities of complete sets of avoidance colourings of order $p$ for small Ramsey numbers. Cardinalities of complete sets of extremal avoidance colourings are underlined. 


\begin{tabular}{c|rrrrrr}
$p$ & $s(4,4)$ & $w(4,4)$ & $u(4,4)$ & $t(4,4)$ & $v(4,4)$ & $r(4,4)$ \\
\hline 1 & 1 & 1 & 1 & 1 & 1 & 1 \\
2 & 2 & 2 & 2 & 2 & 2 & 2 \\
3 & 4 & 4 & 4 & 4 & 4 & 4 \\
4 & 9 & 9 & 9 & 9 & 9 & 9 \\
5 & 24 & 24 & 24 & 24 & 24 & 24 \\
6 & 84 & 84 & 84 & 84 & 84 & 84 \\
7 & 360 & 360 & 360 & 361 & 361 & 362 \\
8 & 2026 & 2026 & 2026 & 2052 & 2052 & 2079 \\
9 & 12915 & 12915 & 12915 & 13775 & 13775 & 14701 \\
10 & 58700 & 58700 & 58700 & 77801 & 77801 & 103706 \\
11 & 56034 & 56034 & 56034 & 180404 & 180404 & 546356 \\
12 & 415 & 415 & 415 & 36080 & 36080 & 1449166 \\
13 & 0 & 0 & 0 & $\underline{8}$ & $\underline{8}$ & 1184231 \\
14 & 0 & 0 & 0 & 0 & 0 & 130816 \\
15 & 0 & 0 & 0 & 0 & 0 & 640 \\
16 & 0 & 0 & 0 & 0 & 0 & 2 \\
17 & 0 & 0 & 0 & 0 & 0 & $\underline{1}$
\end{tabular}

Tab. 4: Cardinalities of complete sets of avoidance colourings of order $p$ for small Ramsey numbers (continued). Cardinalities of complete sets of extremal avoidance colourings are underlined.

the columns labelled " $r(m, n)$ " in Tables 344 (i.e. the cardinalities of complete sets of extremal avoidance colourings for the classical Ramsey numbers) are not our results; they are due to McKay [17], but we reproduce these values here for completeness. We also verified that our computation approach yields the same cardinalities as those reported by McKay for small orders. As final verification mechanism we ensured that the same (extremal) avoidance colourings were obtained for the Ramsey numbers $r(m, n)$, $s(m, n)$ and $t(m, n)$ where $m \neq n$ when interchanging the values of $m$ and $n$.

By considering the zero-entries in these tables, the following two new, off-diagonal Ramsey numbers are established.

Theorem $6 t(6,3)=v(6,3)=17$.

In addition, the following four new, diagonal Ramsey numbers are established in a similar fashion.

Theorem $7 u(4,4)=w(4,4)=13$ and $t(4,4)=v(4,4)=14$.

Taking the six graphs $F_{1}-F_{6}$ in Figure 2(a)-(f) as red subgraphs produces complete sets of extremal avoidance $v(3,5)$-colourings and extremal avoidance $t(3,5)$-colourings. Of these graphs $F_{6}$ as red (blue, respectively) subgraph produces the only extremal avoidance $s(3,5)$-colouring, extremal avoidance $u(3,5)$-colouring and extremal avoidance $w(3,5)$-colouring (extremal avoidance $w(5,3)$-colouring, respectively). Furthermore, taking the graph $F_{7}$ in Figure $2 \mathrm{~g}$ ) as blue subgraph produces the only extremal avoidance $t(5,3)$-colouring and the only extremal avoidance $v(5,3)$-colouring. There are 415 extremal avoidance $s(4,4)$-, $u(4,4)$ - and $w(4,4)$-colourings; these are too numerous to show here, but taking the graph $F_{8}$ in Figure 2(h) as red subgraph produces the extremal avoidance colouring with the smallest number of red edges in all three cases (while the complement of $F_{8}$ as red subgraph produces the extremal 


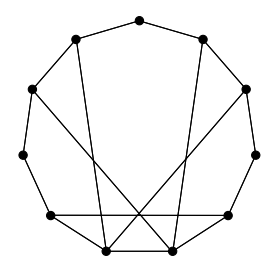

(a) $F_{1}$

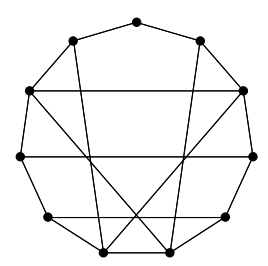

(e) $F_{5}$

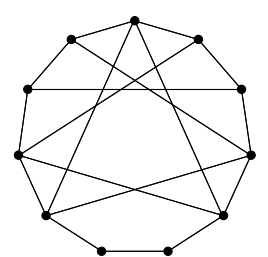

(b) $F_{2}$

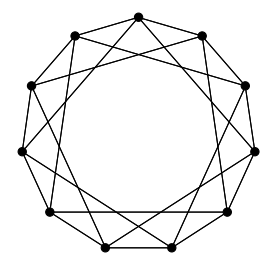

(f) $F_{6}$

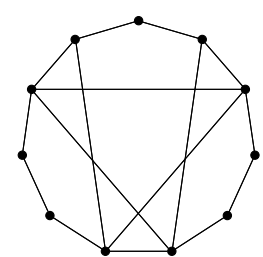

(c) $F_{3}$

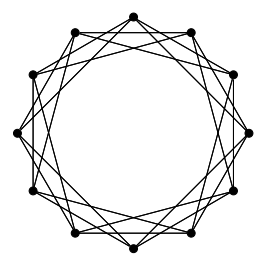

(g) $F_{7}$

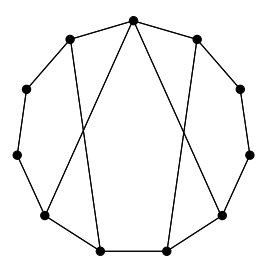

(d) $F_{4}$

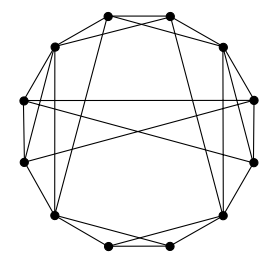

(h) $F_{8}$

Fig. 2: Extremal avoidance graphs of orders 11 and 12.

avoidance colouring with the smallest number of blue edges in all three cases). However, the adjacency matrices of all 415 extremal avoidance $s(4,4)-, u(4,4)$ - and $w(4,4)$-colourings are available online [19].

It is well known that taking the graph $G_{1}$ in Figure 3 a) as red subgraph produces the only extremal avoidance $r(3,5)$-colouring [17]. Furthermore, taking the eight graphs $G_{2}-G_{9}$ in Figure 3 (b)-(i) as red subgraphs produces complete sets of extremal avoidance $t(4,4)$-colourings and extremal avoidance $v(4,4)$-colourings. There are 34 extremal avoidance $t(3,6)$-colourings; these are too numerous to show here, but taking the graph $G_{15}$ in Figure 3 (n) as red subgraph produces the extremal avoidance $t(3,6)$ colouring with the smallest number of red edges, while taking the graph $G_{16}$ in Figure 3 (o) as red subgraph produces the extremal avoidance $t(3,6)$-colouring with the smallest number of blue edges. The adjacency matrices of all 34 extremal avoidance $s(3,6)$-colourings are, however, available online [19]. There are 37 extremal avoidance $v(3,6)$-colourings, namely all 34 extremal avoidance $s(3,6)$-colourings together with the three colourings obtained by taking the graphs $G_{10}-G_{12}$ in Figure $3(\mathrm{j})-(\mathrm{l})$ as red subgraphs. Furthermore, taking the two graphs $G_{13}-G_{14}$ in Figure $3(\mathrm{~m})-(\mathrm{n})$ as red subgraphs [blue subgraphs, respectively] produces complete sets of extremal avoidance $t(3,6)-, u(3,6)$ - and $w(3,6)$-colourings [extremal avoidance $w(6,3)$-colourings, respectively].

In addition, taking the two graphs $H_{1}-H_{2}$ in Figure 4 (a)-(b) as blue subgraphs produces complete sets of extremal avoidance $t(6,3)$ - and $v(6,3)$-colourings. It is known that there are seven extremal avoidance $r(3,6)$-colourings; these colourings are obtained by taking the graphs $H_{3}-H_{9}$ in Figure 4(c)-(i) as red subgraphs. Finally, it is also well known that taking the graph $H_{10}$ in Figure $4(\mathrm{j})$ as red subgraph produces the only extremal avoidance $r(4,4)$-colouring [17].

\section{Conclusion}

In this paper we determined the values of all the outstanding nonclassical Ramsey numbers in Table 1 and described all the extremal avoidance colourings related to the Ramsey numbers in the table, save for the Ramsey number $s(3,7)$. The extremal avoidance colourings of these Ramsey numbers are summarised 


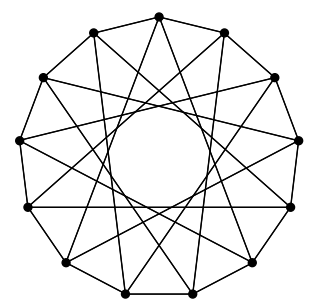

(a) $G_{1}$

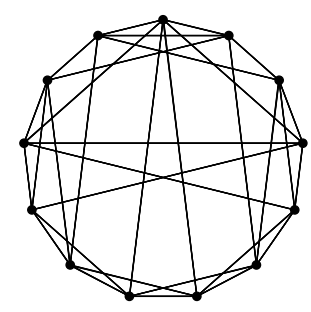

(e) $G_{5}$

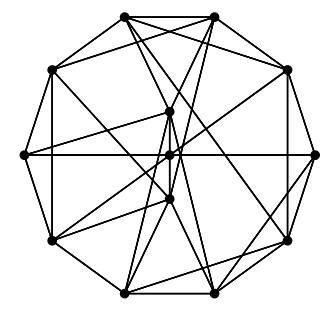

(i) $G_{9}$

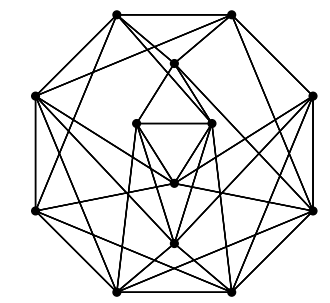

(b) $G_{2}$

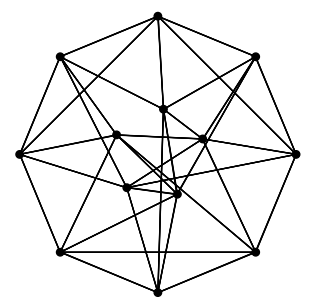

(f) $G_{6}$

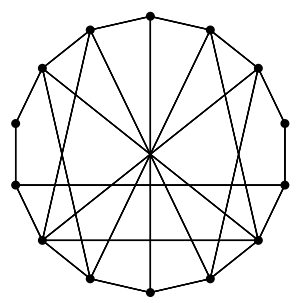

(j) $G_{10}$

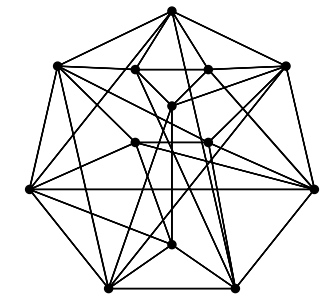

(c) $G_{3}$

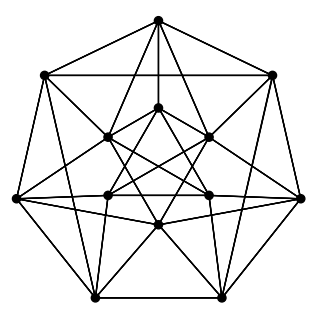

(g) $G_{7}$

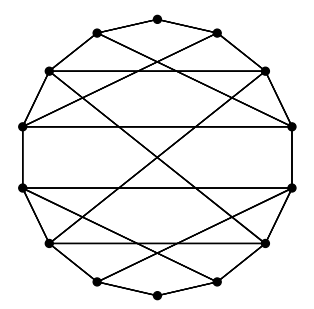

(k) $G_{11}$

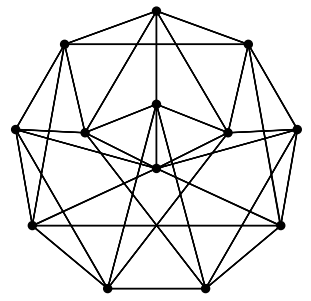

(d) $G_{4}$

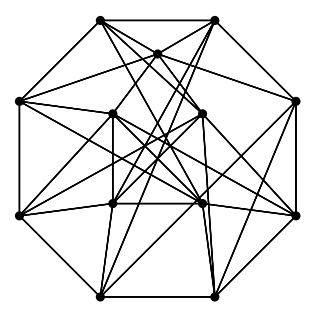

(h) $G_{8}$

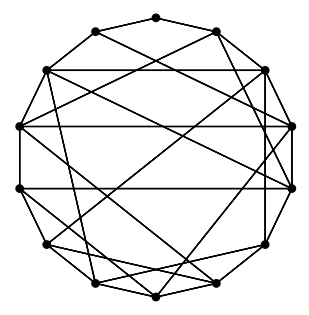

(m) $G_{13}$

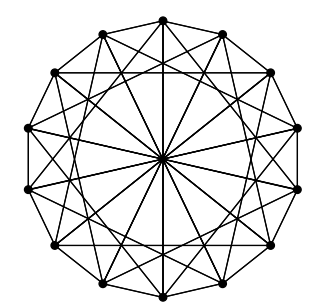

(n) $G_{14}$

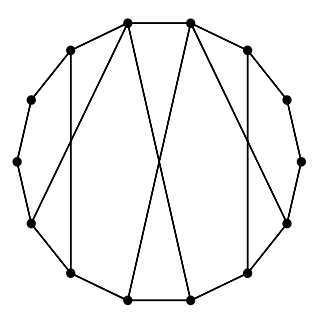

(o) $G_{15}$

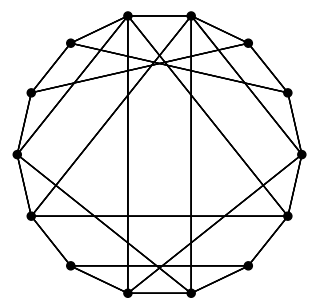

(1) $G_{12}$

Fig. 3: Extremal avoidance graphs of orders 13 and 14. 


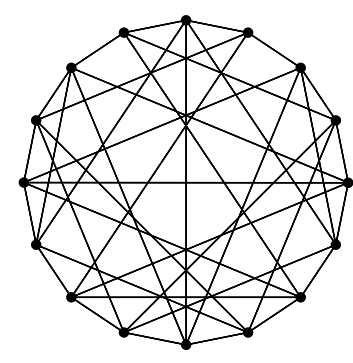

(a) $H_{1}$

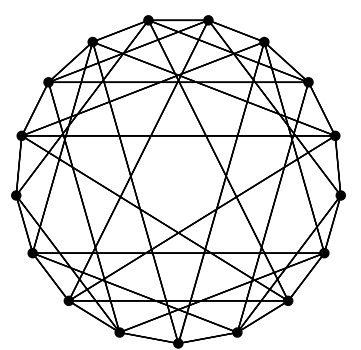

(d) $\mathrm{H}_{4}$

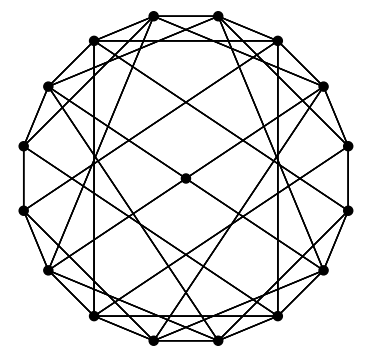

(g) $H_{7}$

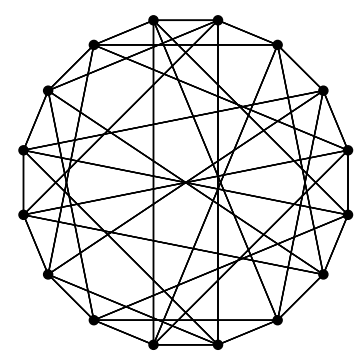

(b) $\mathrm{H}_{2}$

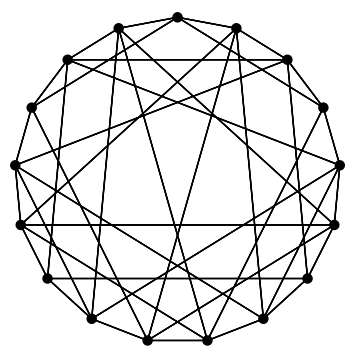

(e) $H_{5}$

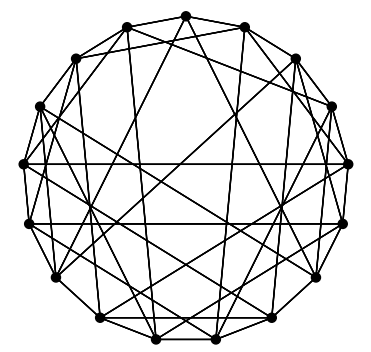

(h) $H_{8}$

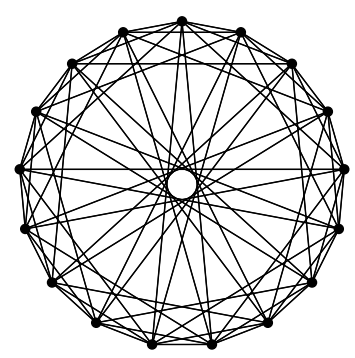

(j) $H_{10}$

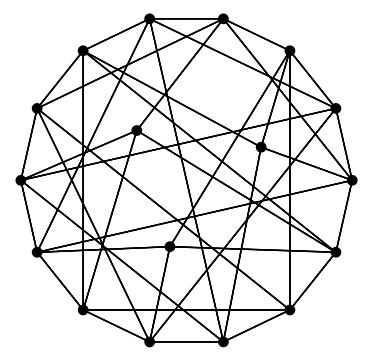

(c) $H_{3}$

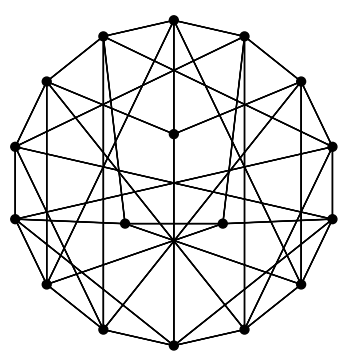

(f) $H_{6}$

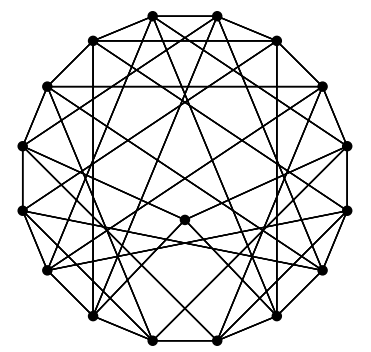

(i) $H_{9}$

Fig. 4: Extremal avoidance graphs of orders 16 and 17. 


\begin{tabular}{|c|c|c|}
\hline $\begin{array}{l}\text { Ramsey } \\
\text { number }\end{array}$ & Source & Extremal avoidance colourings \\
\hline$s(3,3)=6$ & 【1 & 1 Red subgraph $C_{5}$ (and blue $C_{5}$ ) by Theorem 3 \\
\hline$s(3,4)=8$ & [1] & 8 Red subgraphs $E_{1}-E_{8}$ in Figure 1 a)-(h) \\
\hline$s(3,5)=12$ & [1] & 1 Red subgraph $F_{6}$ in Figure 2 f) \\
\hline$s(3,6)=15$ & [2] & 2 Red subgraphs $G_{13}-G_{14}$ in Figure $3(\mathrm{~m})-(\mathrm{n})$ \\
\hline$s(3,7)=18$ & [3, 6 & Unknown \\
\hline$s(4,4)=13$ & [7] & $\begin{array}{l}415 \text { Red subgraphs available online [19]; the smallest is } F_{8} \text { in } \\
\text { Figure } 2(\mathrm{~h}) \text { while the largest is the complement of } F_{8}\end{array}$ \\
\hline$t(3,3)=6$ & {$[5$} & 1 Red subgraph $C_{5}$ (and blue $C_{5}$ ) by Theorem 3 \\
\hline$t(3,4)=9$ & [5] & 2 Red subgraphs $E_{10}-E_{11}$ in Figure $1(\mathrm{j})-(\mathrm{k})$ \\
\hline$t(4,3)=8$ & [5] & 9 Blue subgraphs $E_{1}-E_{9}$ in Figure 1 a)-(i) \\
\hline$t(3,5)=12$ & [5] & 6 Red subgraphs $F_{1}-F_{6}$ in Figure 2 a)-(f) \\
\hline$t(5,3)=13$ & [5] & 1 Blue subgraph $F_{7}$ in Figure 2 g) \\
\hline$t(3,6)=15$ & [11 & $\begin{array}{l}34 \text { Red subgraphs available online [19]; } \\
\text { the smallest and largest are } G_{15}-G_{16} \text { in Figure } 3 \text { (n)-(o) }\end{array}$ \\
\hline$t(6,3)=17$ & New & 2 Blue subgraphs $H_{1}-H_{2}$ in Figure 4 (a)-(b) \\
\hline$t(4,4)=14$ & New & 8 Red subgraphs $G_{2}-G_{9}$ in Figure 3 b)-(i) \\
\hline$u(3,3)=6$ & {$[15$} & 1 Red subgraph $C_{5}$ (and blue $C_{5}$ ) by Theorem 3 \\
\hline$u(3,4)=8$ & [15 & 8 Red subgraphs $E_{1}-E_{8}$ in Figure 1 a)-(h) \\
\hline$u(3,5)=12$ & 15 & 1 Red subgraph $F_{6}$ in Figure 2 f) \\
\hline$u(3,6)=15$ & 15 & 2 Red subgraphs $G_{13}-G_{14}$ in Figure $3(\mathrm{~m})-(\mathrm{n})$ \\
\hline$u(4,4)=13$ & New & $\begin{array}{l}415 \text { Red subgraphs available online [19]; the smallest is } F_{8} \text { in } \\
\text { Figure } 2(\mathrm{~h}) \text { while the largest is the complement of } F_{8}\end{array}$ \\
\hline$v(3,3)=6$ & {$[15$} & 1 Red subgraph $C_{5}$ (and blue $C_{5}$ ) by Theorem 3 \\
\hline$v(3,4)=9$ & [15] & 2 Red subgraphs $E_{10}-E_{11}$ in Figure $1(\mathrm{j})-(\mathrm{k})$ \\
\hline$v(4,3)=8$ & New & 9 Blue subgraphs $E_{1}-E_{9}$ in Figure 1 a)-(i) \\
\hline$v(3,5)=12$ & 15 & 6 Red subgraphs $F_{1}-F_{6}$ in Figure 2 a)-(f) \\
\hline$v(5,3)=13$ & New & 1 Blue subgraph $F_{7}$ in Figure $2(\mathrm{~g})$ \\
\hline$v(3,6)=15$ & 15 & $\begin{array}{l}37 \text { Red subgraphs available online [19]; the } 34 \text { extremal } t(3,6) \text {-avoidance } \\
\text { graphs together with } G_{10}-G_{12} \text { in Figure } 3(\mathrm{j})-(1)\end{array}$ \\
\hline$v(6,3)=17$ & New & 2 Blue subgraphs $H_{1}-H_{2}$ in Figure 4 a)-(b) \\
\hline$v(4,4)=14$ & New & 8 Red subgraphs $G_{2}-G_{9}$ in Figure 3 b)-(i) \\
\hline$w(3,3)=6$ & New & 1 Red subgraph $C_{5}$ (and blue $C_{5}$ ) by Theorem 3 \\
\hline$w(3,4)=8$ & New & 8 Red subgraphs $E_{1}-E_{8}$ in Figure 1 a)-(h) \\
\hline$w(4,3)=8$ & New & 8 Blue subgraphs $E_{1}-E_{8}$ in Figure 1 (a)-(h) \\
\hline$w(3,5)=12$ & New & 1 Red subgraph $F_{6}$ in Figure 2 f) \\
\hline$w(5,3)=12$ & New & 1 Blue subgraph $F_{6}$ in Figure 2 (f) \\
\hline$w(3,6)=15$ & New & 2 Red subgraphs $G_{13}-G_{14}$ in Figure $3(\mathrm{~m})-(\mathrm{n})$ \\
\hline$w(6,3)=15$ & New & 2 Blue subgraphs $G_{13}-G_{14}$ in Figure 3 (m)-(n) \\
\hline$w(4,4)=13$ & New & $\begin{array}{l}415 \text { Red subgraphs available online [19]; the smallest is } F_{8} \text { in } \\
\text { Figure 2(h) while the largest is the complement of } F_{8}\end{array}$ \\
\hline$r(3,3)=6$ & [10] & 1 Red subgraph $C_{5}$ (and blue $C_{5}$ ) by Theorem 3 \\
\hline$r(3,4)=9$ & [10] & 3 Red subgraphs $E_{10}-E_{12}$ in Figure $1(\mathrm{j})-(\mathrm{l})$ \\
\hline$r(3,5)=14$ & [10] & 1 Red subgraph $G_{1}$ in Figure 3 a) \\
\hline$r(3,6)=18$ & [9] & 7 Red subgraphs $H_{3}-H_{9}$ in Figure 4 (c)-(i) \\
\hline$r(4,4)=18$ & [10 & 1 Red subgraph $H_{10}$ in Figure 4 j) \\
\hline
\end{tabular}

Tab. 5: The extremal avoidance colourings associated with the nonclassical Ramsey numbers in Table 1 Corresponding results for the classical Ramsey numbers are also included for completeness. 
in Table 5 and were computed from the avoidance colourings of the corresponding classical Ramsey numbers, which are available online [17]. The reason for not computing the extremal avoidance $s(3,7)$ colourings is that the corresponding colourings are not available for the corresponding classical Ramsey number $r(3,7)$.

\section{Acknowledgements}

The authors thank Martin Kidd who produced the graphics in this paper.

\section{References}

[1] R. Brewster, E. Cockayne, and C. Mynhardt. Irredundant Ramsey numbers for graphs. Journal of Graph Theory, 13:283-290, 1989.

[2] R. Brewster, E. Cockayne, and C. Mynhardt. The irredundant Ramsey number $s(3,6)$. Quaestiones Mathematicae, 13:141-157, 1990.

[3] G. Chen and C. Rousseau. The irredundant Ramsey number $s(3,7)$. Journal of Graph Theory, 19: 263-270, 1995.

[4] E. Cockayne, S. Hedetniemi, and D. Miller. Properties of hereditary hypergrphs and middel graphs. Canadian Mathematics Bulletin, 21:461-468, 1978.

[5] E. Cockayne, J. Hattingh, J. Kok, and C. Mynhardt. Mixed Ramsey numbers and irredundant Turán numbers for graphs. Ars Combinatoria, 29C:57-68, 1990.

[6] E. Cockayne, J. Hattingh, and C. Mynhardt. The irredundant Ramsey number $s(3,7)$. Utilitas Mathematica, 39:145-160, 1991.

[7] E. Cockayne, G. Exoo, J. Hattingh, and C. Mynhardt. The irredundant Ramsey number $s(4,4)$. Utilitas Mathematica, 41:119-128, 1992.

[8] T. Dzido and R. Zakrzewska. The upper domination Ramsey number $u(4,4)$. Discussiones Mathematicae Graph Theory, 26:419-430, 2006.

[9] J. Graver and J. Yackel. Some graph theoretic results associated with Ramsey's theorem. Journal of Combinatorial Theory, 4:125-175, 1968.

[10] R. Greenwood and A. Gleason. Combinatorial relations and chromatic graphs. Canadian Journal of Mathematics, 7:1-7, 1955.

[11] P. Grobler. Irredundant ramsey numbers. Technical report, University of South Africa, Pretoria, 1992.

[12] J. Hattingh. On irredundant Ramsey numbers for graphs. Journal of Graph Theory, 14:437-441, 1990.

[13] T. Haynes, S. hedetniemi, and P. Slater. Fundamentals of domination in graphs. Marcel Dekker, Inc., New York (NY), 1998. 
[14] M. Henning and O. Oellermann. The upper domination Ramsey number $u(3,3,3)$. Discrete Mathematics, 242:103-113, 2002.

[15] M. Henning and O. Oellermann. On upper domination Ramsey numbers of graphs. Discrete Mathematics, 274:125-135, 2004.

[16] B. McKay. Graphs, 1995. URL http://cs.anu.edu.au/ bdm/data/graphs.html,

[17] B. McKay. Ramsey graphs, 1995. URL http://cs.anu.edu.au/ bdm/data/ramsey. html.

[18] O. Oellermann and W. Shreve. Unpublished manuscript.

[19] J. van Vuuren. Repositories, 1995. URL http://www.vuuren.co.za 